\title{
Automatic crystal orientation mapping of Kimberlite nodules using electron back scattered diffraction in the scanning electron microscope
}

\author{
David Mainprice $^{1}$, Guilhem Barruol ${ }^{1}$, Walid Ben Ismaïl ${ }^{1}$ and Geoffrey Lloyd ${ }^{2}$ \\ ${ }^{1}$ CNRS, Laboratoire de Tectonophysique, Université Montpellier II.34095 Montpellier cédex 05, France. email: \\ david@dstu.univ-montp2.fr
}

2 Department of Earth Sciences, The University, Leeds LS2 9JT, United Kingdom.

A new generation of PC-driven scanning electron microscopes (SEM) allows the development of dedicated, low cost systems for automated measurements of electron diffraction patterns over specified sample areas. The technique of electron back scattered diffraction patterns (EBSP) has been available for some time for metallugical specimens. However, minerals (especially rock-forming silicates) differ from metals because they interact with the electron beam and in their microstructural characteristics:

a) Minerals tend to have a low yield of back scattered electrons resulting in diffraction patterns of low intensity above a high background level.

b) Most minerals are insulators which requires a thin carbon coating to reduce electron charge build up which reduces diffraction pattern intensity. Some specimens still charge even when coated because the coating is ruptured due to grain bounday cracks, pores or cleavages. Charging results in a non-flat background for a given beam position.

c) Minerals have low symmetry and their diffraction patterns often display a pseudo-symmetry of higher symmetry, for example orthorhombic olivine has diffraction patterns which have pseudohexagonal symmetry.

d) Rocks tend to be poly-phase with a wide variation in grain size from the centimeter to micron scale, whereas metallugical specimens tend to be monophase of fine uniform grain size.

We have developed an EBSP system to meet the requirements of geological specimens composed of the following elements (see Figure below):

a) A JEOL PC-driven JSM-5600 SEM.

b) A phosphor screen to convert the back scattered electron pattern into photons (light), 
c) A Photonic Science "High Grain" Mono CoolView 768 CCD camera with a high sensitivity to the low intensity light in the wavelength emitted by the screen. The camera combines high sensitivity with the possibility of output at fast video rates.

d) A Matrox PULSAR grabber card which allows the digital diffraction image aquistion at 10 bits (1024 grey levels) at video rates.

e) A system of individual diffraction pattern background correction.

f) A software of EBSP indexation specifically written for low symmetry minerals (Channel+ from HKLsoftware).

f) A system of automatic acquisition of crystal orientations of a fine (using electron beam control) or coarse (using specimen stage control) sample grids under PC supervision using Channel+.
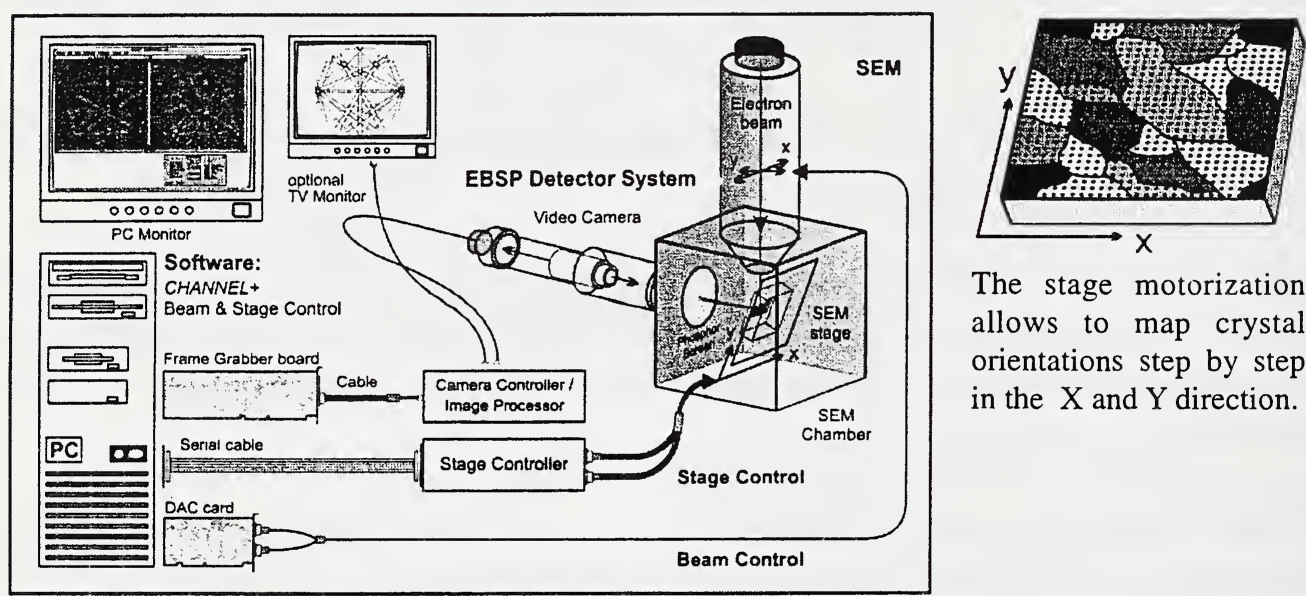

The stage motorization allows to map crystal orientations step by step in the $\mathrm{X}$ and $\mathrm{Y}$ direction.

Experimental setup of the EBSP system (from HKL software)

Kimberlite nodules represent our first test case for our new system. The wide range of grain size, microstructure and mineralogy represents a major challenge for this technique. The first results obtained on PHN1611 have confirmed previous U-stage measurements of crystal preferred orientations and permitted the first automatic crystal orientation maps to be constructed for olivine and orthopyroxene. This sample has a unusual olivine crystal preferred orientation with [010] forming a point maximum parallel to the $\mathrm{Y}$ specimen direction. In fact, the sample is composed of only two crystal orientations which are related by 90 degree rotation about [010] or a 180 degree rotation about an axis normal to [010] in similar way to a twin relationship. The possible origins of this olivine crystal preferred orientation will be dicussed in the light of these new observations. 